\title{
History Matching: Towards Geologically Reasonable Models
}

\author{
MeInikova, Yulia; Cordua, Knud Skou; Mosegaard, Klaus
}

Publication date:

2012

Document Version

Publisher's PDF, also known as Version of record

Link back to DTU Orbit

Citation (APA):

Melnikova, Y., Cordua, K. S., \& Mosegaard, K. (2012). History Matching: Towards Geologically Reasonable Models. Abstract from EAGE Integrated Reservoir Modelling: Are we doing it right? , Dubai, United Arab Emirates.

\section{General rights}

Copyright and moral rights for the publications made accessible in the public portal are retained by the authors and/or other copyright owners and it is a condition of accessing publications that users recognise and abide by the legal requirements associated with these rights.

- Users may download and print one copy of any publication from the public portal for the purpose of private study or research.

- You may not further distribute the material or use it for any profit-making activity or commercial gain

- You may freely distribute the URL identifying the publication in the public portal

If you believe that this document breaches copyright please contact us providing details, and we will remove access to the work immediately and investigate your claim. 


\section{RM25}

\section{History Matching: Towards Geologically Reasonable Models}

Y. Melnikova* (Technical University of Denmark), K.S. Cordua (Technical
University of Denmark) \& K. Mosegaard (Technical University of Denmark)

\section{SUMMARY}

This work focuses on the development of a new method for history matching problem that through a deterministic search finds a geologically feasible solution. Complex geology is taken into account evaluating multiple point statistics from earth model prototypes - training images.

Further a function that measures similarity between statistics of a training image and statistics of any smooth model is introduced and its analytical gradient is computed. This allows us to apply any gradientbased method to history matching problem and guide a solution until it satisfies both production data and complexity of a prior model with desired accuracy. As a consequence of the approach, we sufficiently decrease the amount of forward simulations needed to resolve historical data and prior information. 


\section{Introduction}

History matching is an essential part of reservoir characterization process. Reliable reservoir models must fit production history and feature expected geology. Therefore geological a priori information should be included in the estimation of reservoir parameters. Due to the high computational cost of forward simulations (reservoir simulator runs) use of Monte-Carlo techniques can be unfeasible.

A fast compromise solution would be to find an approximation of the maximum a posteriori solution (MAP). To succeed in this task the probability of the model to resolve geological features (prior probability) must be estimated. Recently Lange et al. (2012) suggested the Frequency Matching (FM) method for solving inverse problems by use of geologically realistic prior information. In the FM approach the a priori information takes the form of multiple-point statistics learned from reservoir geological prototypes - training images (e.g. Guardiano and Srivastava 1993). The attractiveness of the FM method lies in its ability to quantify the prior probability of the proposed model and hence iteratively guide the model towards the maximum a posteriori solution. The FM method solves a combinatorial optimization problem, perturbing the model in a discrete manner until it explains both production data and a priori information. In practice, this requires a lot of forward simulations and can be impractical for solving history matching problems.

While following the philosophy of the Frequency Matching method, we suggest a differentiable expression for a complex prior, so that, as a result, the approximation of the MAP solution can be found by gradient-based techniques with much fewer forward simulations required.

\section{Methodology}

We suggest a gradient-based method for obtaining geologically feasible solutions of history matching problem. The algorithm integrates production data and complex geological a priori information into a single objective function. Importantly, we propose a differentiable formulation of a priori information.

As a priori information, we use multiple point statistics derived from training images, which characterizes the expected spatial distribution of the sought physical property, for instance, permeability. Similar to Lange et al. (2012) we define an optimization problem, i.e. to minimize:

$$
\mathrm{O}(\mathbf{m})=\frac{1}{2}\left\|d^{\mathrm{obs}}-g(\mathbf{m})\right\|_{C_{d}}^{2}+f(\mathbf{m}, \mathrm{TI})
$$

Reservoir parameters $\mathbf{m}$ are then inferred by minimizing two misfits: 1) between observed production data $d^{\text {obs }}$ and reservoir response $g(\mathbf{m})$ and 2) between statistics of the model (test image) $\mathbf{m}$ and statistics of the training image TI. One way to collect the statistics is to apply a scanning template to an image and compute the frequency distribution of the event defined by the chosen template (Lange et al., 2012). The result will be the histogram that describes the image uniquely. The distance (defined in some sense) between the histogram of the training image and one of the test image estimates their statistical similarity.

The challenge in the gradient-based approach is to define a differentiable similarity measure between the continuous image $\mathbf{m}$ and the discrete training image TI. Keeping the idea of histogram in mind, we first define the similarity function $h_{i j}$ between a continuous pattern $i$ and a discrete pattern $j$, using the normalized Euclidian distance $d_{i j}$ between their pixels values:

$$
h_{i j}=\frac{1}{\left(1+A d_{i j}^{k}\right)^{p}}
$$


Here $A, k, p$ are adjustable parameters. Then the pseudo-histogram is constructed calculating the "contributions" $h_{i j}$ of patterns in the image to all possible discrete patterns. The number of the histogram bins is equal to the number of all possible discrete patterns, i.e. $c^{N_{\text {pat }}}$, where $C$ is the number of categories in the training image and $N_{p a t}$ is the number of pixels in the pattern.

In (1) the function $f(\mathbf{m}, \mathrm{TI})$ is the L2 norm of the difference between the pseudo-histograms of the training image $\mathrm{TI}$ and the test image $\mathbf{m}$.

For solving (1) we chose the unconstrained implementation of the LBFGS method (Zhu et al. 1997), which is known to be efficient for history matching problems (Oliver et al. 2008). To use unconstrained optimization we applied the logarithmic scaling of reservoir parameters proposed in Gao and Reynolds (2006):

$x_{i}=\ln \underset{\square}{\square m_{i}-m^{\text {ow }}-m^{i}} \sqsubset$

Here $i=1, \ldots, n$, where $n$ is the number of pixels in the test image $\mathbf{m}, m^{\text {low }}$ and $m^{u p}$ are the lower and upper scaling boundaries respectively. Global criterion method (Marler and Arora 2004) was used to combine the data misfit and prior terms into one objective function. This yielded to the final look of the objective function:

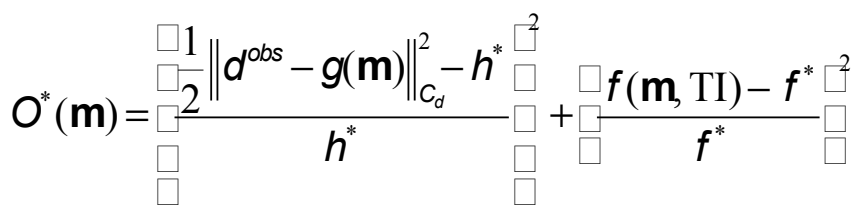

Here $h^{*}$ and $f^{*}$ are the target values for data and prior misfits respectively. For forward simulations E300 reservoir simulator was used (Schlumberger GeoQuest 2009). The gradient of the data misfit term in (4) was evaluated using the adjoint calculation implemented in E300. The gradient of the prior term in (4) was calculated analytically.

As in any gradient-based technique, solution and convergence properties of the suggested method are strongly dependent on the initial guess and quality of the production and statistical data. In case of a poor choice of the template size geological features cannot be reproduced. However, large amount of data may compensate for the lack of statistical information. In the numerical example below we will see how sufficient wells coverage yields the correct length of geological features, while a priori information resolves their width in agreement with training image.

\section{Numerical example}

In the test study we aim at reconstructing permeability field of a $2 \mathrm{D}$ synthetic oil reservoir of 49x49x1cells. The true permeability and wells (9 injectors, triangles and 9 producers, circles) are shown in Figure 1. Training image of 200x200 pixels (Figure 2) has two categories and features highly permeable channels of $10000 \mathrm{mD}$ and $500 \mathrm{mD}$ background. Notice the scaling boundaries of $450 \mathrm{mD}$ for $m^{\text {ow }}$ and $10500 \mathrm{mD}$ for $m^{\mu p}$. Production data were generated by running a forward simulation with the true permeability model and adding $5 \%$ of Gaussian noise. Specifically, the reservoir was in production for 210 days and the data were collected every 30 days. For history matching we used BHP values from the injectors and oil rates from the producers (126 measurements in total).

A priori information was collected applying a 1D-scanning template of 10 pixels in vertical direction. We let the template to take care about the width of the channels, while the production data assure the horizontal continuity. 
While parameters $A, k, p$, in (2) are empirical, such values as 100,2 and -1 respectively provide the optimal quality of the reconstructed image and may serve as a general recommendation.

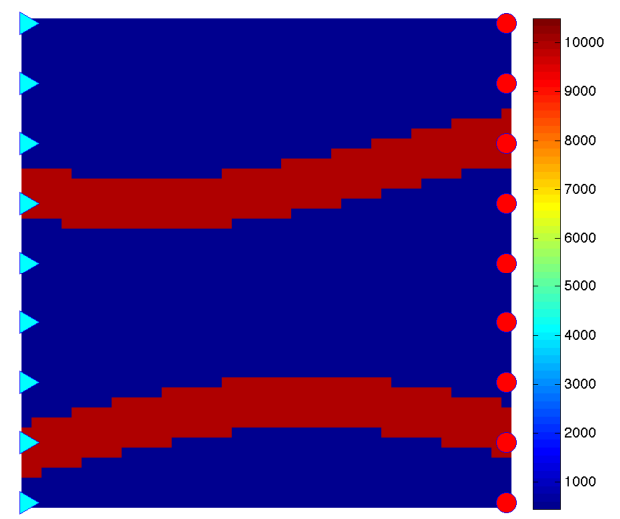

Figure 1 True permeability model, $49 \times 49$ pixels.

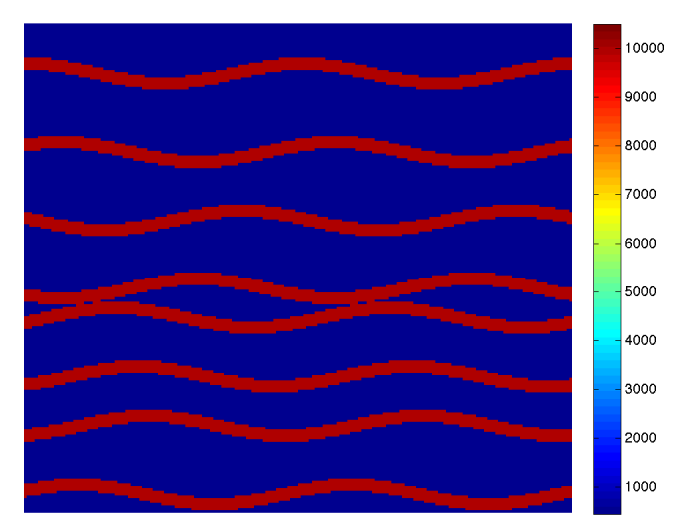

The initial model (see Figure 3) gives the data misfit of the order of $10^{5}$ and the histograms misfit - of $10^{-1}$. In the optimization framework given by (4), we set the target values as 20 and 0.005 for the data and the histograms misfits respectively.

Figure 4 shows the solution at the $97^{\text {th }}$ iteration. Visual inspection tells us that geological features were successfully reproduced. Additionally, the expected order of $10^{-3}$ in the histograms misfit was achieved. The production data were resolved well, obtaining the data misfit equal to 52 (expected $\approx N / 2=63$, where $\mathrm{N}$ is the number of measurements, see, e.g., Oliver et al. (2008)).

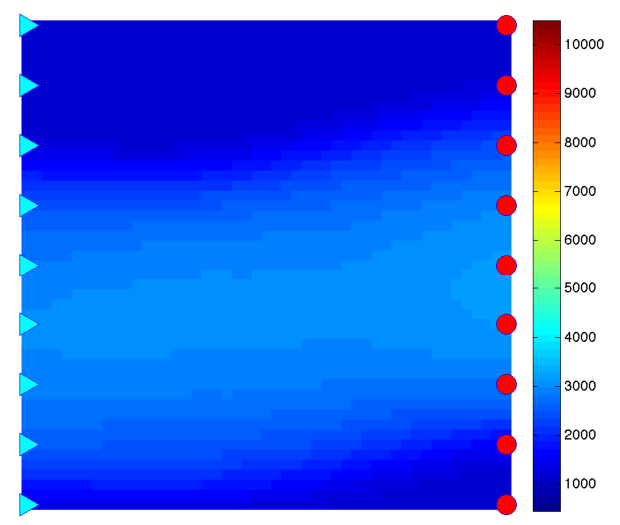

Figure 3 Initial permeability model.

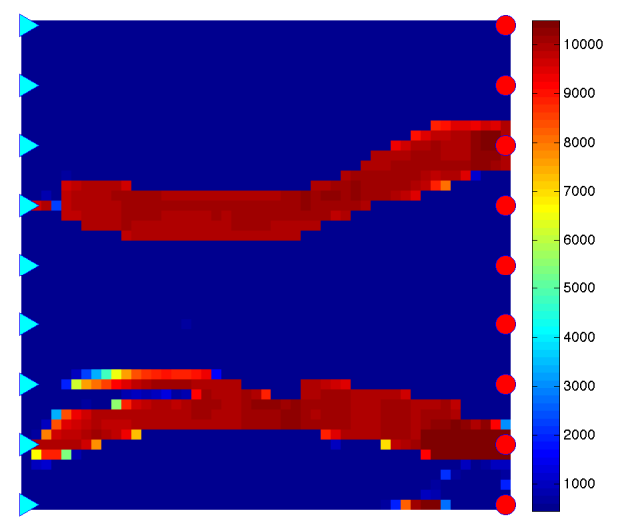

Figure 4 Solution, $97^{\text {th }}$ iteration.

Figure 5 demonstrates history matching for injector 4 and producer 3 (wells are numbered starting from the top). 

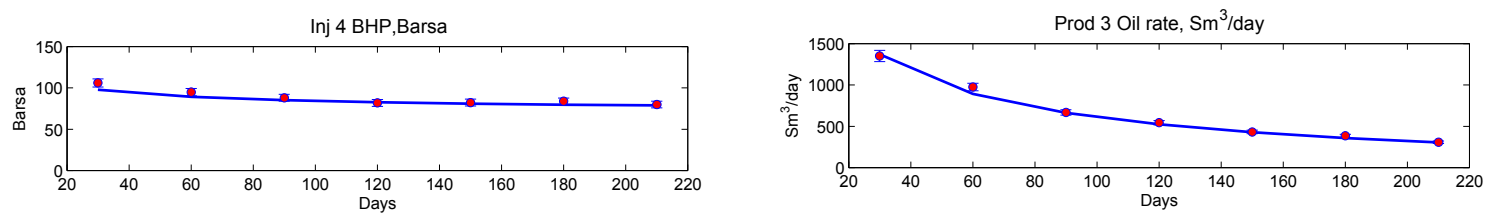

Figure 5 History matching: observed data (red circles) with error bars and solution response (blue line).

\section{Conclusions}

The proposed approach allows us to solve history matching problem by gradient-based optimization techniques, conserving geological realism of the solution. The differentiable formulation scales down the amount of required forward simulations and can be a valuable approach in modern reservoir management techniques as, for instance, in closed-loop optimization. Besides, the ability to quantify prior probability of history-matched reservoir models allows us to control the quality of reservoir characterization choosing the most reliable solutions.

\section{References}

Gao, G. and Reynolds, A.C. [2006] An improved implementation of the LBFGS algorithm for automatic history matching. SPE Journal, 11(1), 5-17.

Guardiano, F. and Srivastava, R. [1993] Multivariate geostatistics: Beyond bivariate moments. Geostatistics Troia, 92(1), 133-144.

Lange, K., Frydendall, J., Cordua, K.S., Hansen, T.M, Melnikova, Y. and Mosegaard, K. [2012] A frequency matching method: solving inverse problems by use of geologically realistic prior information. Accepted to Mathematical Geosciences.

Marler, R.T. and Arora, J.S. [2004] Survey of multi-objective optimization methods for engineering. Structural and Multidisciplinary Optimization, 26(6), 369-395.

Oliver, D.S., Reynolds, A.C., Liu, N. [2008] Petroleum reservoir characterization and history matching. Cambridge University Press, New York.

Schlumberger GeoQuest [2009] ECLIPSE reservoir simulator, Technical description. Houston, TX.

Zhu, C., Byrd, R.H., Lu, P. and Nocedal, J. [1997] L-BFGS-B: Fortran subroutines for large-scale bound-constrained optimization. ACM Transactions on Mathematical Software (TOMS), 23(4), 550560 . 